\title{
RELACIÓN DE LA CONCENTRACIÓN Y FRECUENCIA DE APLICACIÓN DE OZONO CON EL NIVEL DE DAÑo DE LA SIGATOKA NEGRA EN BANANO. DISEÑO DE UN PROTOCOLO DE RIEGO CON AGUA OZONIFICADA
}

\author{
CONCENTRATION RATIO AND FREQUENCY OF APPLICATION \\ LEVEL OZONE DAMAGE BLACK SIGATOKA IN BANANAS. \\ DESIGN OF A PROTOCOL IRRIGATION WITH OZONATED WATER
}

\section{ÁNGEL LLERENA HIDALGO', RAFAEL CASTAÑO OLIV0², CRISTÓBaL JOAQUíN AGUIRRE ${ }^{3}$}

\footnotetext{
Facultad Técnica para el Desarrollo, Universidad Católica de Santiago de Guayaquil. angel.llerena@cu.ucsg.edu.ec

Facultad Técnica para el Desarrollo, Universidad Católica de Santiago de Guayaquil. rafael.castano@cu.ucsg.edu.ec

3 Facultad Técnica para el Desarrollo, Universidad Católica de Santiago de Guayaquil.
}

\section{RESUMEN}

Se estudió el efecto de aplicación de ozono disuelto en agua en el control de Sigatoka Negra en el cultivo de banano, en dos zonas agrícolas con condiciones edafoclimáticas diferentes, una en el cantón Baba y otra en el cantón Jujan. Se hicieron evaluaciones, en dos periodos climatológicos (invierno y verano) de marzo a noviembre del 2014, combinadas con las prácticas culturales tradicionales de deshoja, deshija, fertilización, y el monitoreo, utilizando la escala de signos visuales de 6 puntos de Stover y el método del preaviso temprano. Las variables evaluadas fueron: diámetro del fuste (D/F), hojas por planta (H/P), hoja más joven enferma (HMJE) y porcentaje ponderado de infección (PPI), para cada frecuencia de aplicación evaluada. La variable diámetro del fuste no tuvo diferencia significativa, el número de hojas, $\mathrm{H} / \mathrm{P}$, en la mayoría de los tratamientos se inició con un mínimo de 3 hojas hasta un máximo de 14 hojas sanas o partes de ella a cosecha, la HMJE, en la mayoría de los tratamientos se inició en la hoja número 3 y el PPI, en todos los tratamientos se inició con un mínimo de 0,10 a un máximo de 2,1\%. En los testigos los valores para las variables evaluadas fue de 3 a 9 hojas por planta, hoja más joven enferma la número 2 y el porcentaje de infección varió entre 0,3 a 2,6\%. Estos valores del PPI, en todos los tratamientos, son equivalentes al grado 2 de la escala de Stover (menores al 5\%). Se utilizó un diseño experimental de bloques completamente al azar (BCA), con cuatro repeticiones. Se estableció la relación existente entre la concentración y frecuencia de aplicación de ozono con el nivel de daño de la Sigatoka Negra. Se utilizaron las comparaciones por contrastes ortogonales para evaluar las frecuencias de aplicación con la dosis utilizada, con lo cual se logró un control total de la infección, cosechándose una fruta de calidad y un promedio de 11 hojas a la cosecha.

PALABRAS CLAVE: ozono, control manejo Sigatoka negra, banano.

\section{ABSTRACT}

The different frequencies of ozone application for the control of black Sigatoka in banana cultivation in two agricultural areas with different soil and climatic conditions, in the canton Baba and another in the canton Jujan were evaluated. The main objective was to establish the relationship between concentration and frequency of application Ozone Damage Level Black Sigatoka in Banana and Irrigation Design a protocol with ozonated water to generate a management program based on dissolved ozone in water, applied with a knapsack sprayer motor, to control the disease, and to transfer the methodology to other banana sector.Evaluations were made in two climatic periods (winter and summer) from march to november 2014, combined with traditional cultural practices defoliated, deshija, fertilization, and monitoring, using the scale of visual signs 6 points Stover and early notice of the method. The variables evaluated were: stem diameter (D / F), leaves per plant (H / P), youngest diseased leaf (YDL) and weighted percentage of infection (PPI) for each frequency of application evaluated. The variable stem diameter had no significant difference, number of leaves, $\mathrm{H} / \mathrm{P}$, in most treatments started with a minimum of 3 sheets up to 14 healthy leaves or parts of it to harvest, YDL, in most treatments began in the number 3 and the PPI sheet, all treatments started with a minimum of 0.10 to a maximum of 2.1\%. In the control values for the variables was evaluated 3-9 leaves per plant, youngest diseased leaf number 2 and the infection rate ranged from 0.3 to $2.6 \%$. PPI these values, across all treatments, are equivalent to grade 2 Stover scale (less than 5\%). The experimental design was randomized complete block (BCA), with four replications. Comparisons were used by orthogonal contrasts to assess the frequency of application dose used for the control of black Sigatoka, which total control of infection were harvested fruit of excellent quality and an average of 11 leaves was achieved harvest.

KEYWORDS: ozone, control black Sigatoka management, bananas. 
INTRODUCCIÓN

La enfermedad ocasionada por la Sigatoka negra, $M$. fijiensis, en la planta de banano se ha dispersado por todo el continente americano y el Caribe. En los diferentes países han ocurrido severas epidemias, que han obligado a intensificar las medidas de combate y han puesto de manifiesto la necesidad de estrategias de manejo integrado de la enfermedad, para mitigar su impacto negativo (Guzmán y Villalta, 2006)

El M. fijiensis se reproduce en forma asexual y sexual. La reproducción asexual se presenta en lesiones jóvenes de la enfermedad (estrías 2 y 3 y el primer estadio de mancha). Los conidios aparecen en conidióforos sencillos que emergen de los estomas, principalmente por la superficie abaxial de las hojas. Los conidios se dispersan por el salpique de la lluvia y se asocian con la diseminación de la enfermedad acorta distancia. La fase sexual, de mayor importancia en el desarrollo de la enfermedad, se produce en las lesiones maduras, en estructuras denominadas pseudotecios, en cuyo interior se encuentran las ascosporas, las cuales son liberadas al ambiente en períodos de alta humedad para ser dispersadas hasta largas distancias por las corrientes de aire. (Stover,1980), (Martínez, Villalta, Soto, Murillo \& Guzmán, 2011).

El control químico y la selección de plantas resistentes continúan siendo las únicas estrategias, por excelencia, para combatir la Sigatoka negra según Riveros y Lepoivre (1998). Para los pequeños agricultores, los clones resistentes o tolerantes serían las medidas de control mejor adaptadas a su formación técnica y al contexto socio económico de este cultivo. Hoy día el combate químico es la alternativa más usada para hacerle frente, aunque trae problemas colaterales (Orozco-Santos, 1998).

El combate químico es la principal herramienta para el manejo de la Sigatoka negra. Se realiza mediante la aplicación alterna y en mezcla de fungicidas protectores y sistémicos. Los fungicidas protectores son de acción multisitio (bajo o nulo riesgo de resistencia) y se incluyen en este grupo el Mancozeb y el Clorotalonil. Los sistémicos son de acción sitio-específico (moderadora alto riesgo de resistencia) e incluyen fungicidas de grupos como benzimidazoles, aminas, triazoles, estrobirulinas y anilino pirimidinas. Además, se encuentran en proceso de registro nuevos fungicidas sistémicos de dos grupos químicos nunca antes utilizados en banano: carboxamidas y guanidinas. $M$. fijiensis ha desarrollado resistencia a los benzi- midazoles, triazoles y estrobirulinas, lo cual ha reducido su eficacia en campo y limitado su uso (Martínez y Guzmán, 2010).

Según Escudero y Rondón (1996) en la actualidad se ha intensificado el uso de fungicidas protectantes en todas las áreas productoras, realizando aplicaciones periódicas cada siete a 12 días. Con la implementación de los programas de protectantes basado en fungicidas Mancozeb, se requieren aplicaciones semanales durante la época de lluvia y cada 10-14 días durante la época de seca, que anualmente serían 30-35 aplicaciones (Orozco-Santos, Farías, Manzo, Sánchez y Guzmán, 2001).

La evaluación de nuevas moléculas de fungicidas sin o pocos efectos nocivos al ambiente y salud humana son prioritarios para la búsqueda de nuevas alternativas de manejo de Sigatoka negra, dentro de este grupo de fungicidas se encuentra el Azoxistrobin mencionado anteriormente, que es seguro desde el punto de vista ambiental (Orozco-Santos et al., 2001).

Zuluaga, Patiño y Collazos (2007) evaluaron en condiciones de campo en la zona bananera de Urabá-Colombia, el efecto de inductores de resistencia y bacterias quitinolíticas sobre el desarrollo de la Sigatoka negra en plantas de banano del cultivar Gran Enano. Como inductores de resistencia se utilizaron acibenzolar-s-metil (ASM) $(20 \mathrm{~cm} 3$ • ha-1 i.a.) y ácido salicílico (AS) (20 cm3 - ha-1 i.a). La aplicación de ASM en rotación y en mezcla con fungicidas convencionales presentó un control de la enfermedad hasta un 50\% mayor al obtenido con el control convencional. Los tratamientos, que incluyeron AS en rotación y mezcla con fungicidas convencionales, al igual que el producto Biofun ${ }^{\circledR}$ en rotación y en mezcla con ASM, mostraron un control de la enfermedad estadísticamente igual al obtenido con el control convencional, lo cual reveló una reducción de 46 - 100\% de la cantidad de fungicidas convencionales usados en el control de la Sigatoka negra.

Actualmente el control de la Sigatoka negra, se centra en la aplicación de fungicidas químicos; sin embargo, el patógeno ha desarrollado resistencia, aumentando los costos de control, el impacto negativo sobre el ambiente y la exigencia de los consumidores por una fruta cada vez más libre del uso de plaguicidas. La aplicación de sustratos foliares con base en quitina coloidal, harina de cebada como fuente del polisacárido glucano, urea y una solución mineral base, diseñados para fomentar poblaciones de bacterias quitinolíticas y glucanolíticas de ocu- 
rrencia natural, mostró una reducción entre un 43 y $46 \%$ en el número de ciclos de fungicidas convencionales, al ser aplicados en rotación con estos últimos, con relación al sistema convencional basado en la aplicación de fungicidas. (Patiño, Bustamante y Salazar, 2007)

Tumbaco y Jiménez (2011) emplearon un extracto de "Melaleuca alternifolia" como biofungicida para el control de la enfermedad causada por el hongo Mycosphaerella fijensis que afecta a todas las variedades de musáceas, asociándolo con factores tales como el número de aplicaciones, el solvente más apropiado, la tecnología utilizada y la concentración del extracto. Elobjetivo principaldelestudio fue monitorear el agente causal de la enfermedad Sigatoka negra en banano mediante evaluaciones semanales en campo. El efecto del extracto sobre el patógeno se evaluó semanalmente en campo con la metodología de Stover. Los resultados mostraron que las concentraciones del extracto de Melaleuca Alternifolia ejercieron un control de Micosphaerella Fijiensis en campo en las tres provincias estudiadas y demostró propiedades fungicidas al disminuir la severidad de la enfermedad durante el periodo de tiempo en las cuales se realizaron las observaciones en campo.

El ozono, (O3), es una forma alotrópica del oxígeno molecular (O2) que se origina en las capas altas de la atmósfera por la radiación UV de los rayos solares que reacciona con dicha molécula de oxígeno, disociándola y la reacción posterior de los iones formados con otras moléculas de oxígeno; está presente como un constituyente gaseoso natural en las capas altas de la atmósfera y representa el 0,0001\% de su composición total (Arencibia, Leyva, Collymore \& Araujo, 2006). En las capas más bajas de la atmósfera se origina a partir de las descargas eléctricas.

El ozono no puede ser almacenado debido a su gran inestabilidad química a presión y temperatura ambiente; esta situación obliga a los investigadores a disponer de un equipo generador del gas de tal manera que para cada experimento sea necesario obtenerlo en el momento. Debe considerarse que el ozono, respecto al oxígeno, es casi doce veces más soluble en agua.

\section{MATERIALES Y MÉTODOS}

Se empleó un equipo generador de ozono construido por los investigadores de acuerdo a lo planteado por Beutelspacher y Calderón (2005). Las especificaciones técnicas del equipo son:

1. Voltaje de entrada: $100 \mathrm{~V}-140 \mathrm{~V} 50 / 60 \mathrm{~Hz}$

2. Voltaje de salida: $\quad 5.0 \mathrm{KV} \pm 0.5 \mathrm{KV}$
3. Salida de ozono: $20 \mathrm{~g} / \mathrm{h}$ de ozono más (cuando la entrada voltaje es 110V/50Hz)

4. Entrada de corriente: menos de 2.2A

5. Entrada de energía: menos de $240 \mathrm{~W}$

6. Temperatura de trabajo: $-10^{\circ} \mathrm{C}-60^{\circ} \mathrm{C}$

7. Humedad ambiental: $35 \%-85 \% \mathrm{RH}$

8. Temperatura de almacenamiento: $-20^{\circ} \mathrm{C}-$ $70^{\circ} \mathrm{C}$

9. Temperatura y humedad prueba: cuando que está funcionando el generador, a temperatura y humedad constantes; la caja cuya temperatura es de $45^{\circ} \mathrm{C} \pm 3^{\circ} \mathrm{Cy}$ la humedad de $90 \% \pm 5 \%$ (todas las actuaciones son normales después de 72 horas).

10. Prueba da alta temperatura: cuando está en funcionamiento el generador de ozono, a la temperatura de prueba; temperatura de la caja es de $65^{\circ} \mathrm{C} \pm 5^{\circ} \mathrm{C}$, (todas las actuaciones son normales después de 72 horas).

11. Prueba de baja temperatura: cuando el generador de ozono está trabajando, se puso en la caja de prueba de baja temperatura $-20^{\circ} \mathrm{C} \pm 5^{\circ} \mathrm{C}$, todas las actuaciones son normales después de 72 horas.

12. Alta y baja temperatura (prueba de impacto): cuando está en funcionamiento el generador de ozono, se puso en la caja de prueba de baja temperatura $-10^{\circ} \mathrm{C} \pm 5^{\circ} \mathrm{C}$ durante 1 hora, luego dejó de funcionar; el generador del ozono trabaja durante 1 hora a la temperatura normal. A continuación, se coloca en la caja de prueba de alta temperatura $65^{\circ} \mathrm{C} \pm 5^{\circ} \mathrm{C}$ a trabajar durante 1 hora. Todas las actuaciones son normales.

13. Prueba de baja temperatura almacenamiento: poner el generador de ozono en la caja de prueba de baja temperatura cuya temperatura es de $-30^{\circ} \mathrm{C} \pm 5^{\circ} \mathrm{C}$ por 72 horas y luego prueba. Todas las actuaciones son normales.

14. Prueba de alta temperatura almacenamiento: poner el generador de ozono en la caja de prueba de alta temperatura cuya temperatura es de $75^{\circ} \mathrm{C} \pm 5^{\circ} \mathrm{C}$ por 72 horas y luego prueba. Todas las actuaciones son normales.

15. Prueba gota: el generador de ozono el paquete en la caja de cartón normal y soltar la caja de cartón de $200 \mathrm{~cm}$ por encima del suelo. Haga la prueba de gota para cada lado de la caja de cartón. Todas las actuaciones son normales. 
16. Generador de espacio de descarga del ozono es inferior a $0,4 \mathrm{~mm}$; el tiempo de trabajo regular del generador es de más 10000 horas.

Una vez construido el equipo se procedió a realizar las siguientes actividades:

- Calibración del equipo para medir la dosis adecuada en partes por millón (ppm)

- Identificación del campo donde se va a realizar el ensayo, tomas de muestra de suelo y de agua para sus análisis respectivos.

- Delineamiento experimental de las parcelas.

\section{CALIBRACIÓN DEL EQUIPO}

Se realizaron cuatro pruebas, de cinco repeticiones cada una, variando el tiempo de ozonificación y manteniendo constantes los demás parámetros, como se aprecia a continuación:

Primera prueba, ozonificación del agua durante 5 minutos

- 1 litro de agua.

- Ozonizar el agua durante 5 minutos, con 5 libras de oxígeno.

- Agregar agua

- Encerar la ampolla.

- 5 gotas del activador A-7400.

- Agregar $25 \mathrm{ml}$ de agua ozonizada

- Con la ampolla se la mezcla y se rompe la ampolla (como consecuencia cambia a color morado).

- Agitar la ampolla durante un minuto.

- Colocar la ampolla en el medidor.

- Después de 20 minutos se volvió a realizar la prueba con la misma agua ozonizada.

RESULTADO: 0,11PPM DE OZONO EN AGUA (PROMEDIO) Segunda prueba, ozonificación del agua durante 3 minutos

- En un litro de agua.

- Ozonizar el agua durante 3 minutos, con 5 libras de oxigeno.

- Encerar la ampolla.

- 5 gotas del activador A-7400.

- $25 \mathrm{ml}$ de agua ozonizada.

- Con la ampolla se la mezcla y se rompe la ampolla (como consecuencia cambia a color morado).

- Se debe agitar la ampolla durante un minuto.

- Colocar la ampolla en el medidor.
RESULTADO: 0,64 PPM DE OZONO EN AGUA (PROMEDIO)

Tercera prueba, ozonificación del agua durante 7 minutos.

- En un litro de agua.

- Ozonizar el agua durante 7 minutos, con 5 libras de oxígeno.

- Encerar la ampolla.

- 5 gotas del activador A-7400.

- Más 25 ml de agua ozonizada.

- Con la ampolla se la mezcla y se rompe la ampolla (como consecuencia cambia a color morado).

- Se debe agitar la ampolla durante un minuto.

- Colocar la ampolla en el medidor.

RESULTADO: 1,89 PPM DE OZONO EN AGUA (PROMEDIO)

Cuarta prueba, ozonificación del agua durante 5 minutos.

- En un litro de agua.

- Ozonizar el agua durante 5 minutos, con 5 libras de oxigeno.

- Encerar la ampolla.

- 5 gotas del activador A-7400.

- Más 25 ml de agua ozonizada.

- Con la ampolla se la mezcla y se rompe la ampolla (como consecuencia cambia a color morado).

- Se debe agitar la ampolla durante un minuto.

- Colocar la ampolla en el medidor.

RESULTADO: 2,08 PPM DE OZONO EN AGUA (PROMEDIO)

Se demostró que el tiempo óptimo de ozonificación del agua para riego debe ser de 5 minutos.

Determinación de la concentración óptima de ozono en ppm.

1. Para determinar la dosis óptima de ozono se hicieron varias pruebas de campo inyectando oxígeno al generador de ozono de 20 gramos por hora, lo cual da una relación de 5 litros por minuto.

2. El caudal fue de cuatro galones por minuto equivalente a una dosis de cuatro partes por millón (ppm).

3. La inyección del ozono al agua se la realizó a través de un venture $\operatorname{ADF} 3 / 4$ resistente al ozono.

4. La concentración de ozono fue medida con un equipo chemetrics modelo i-2019 especializado para la medición de ozono en partes de millón (ppm).

5. Se realizaron varias pruebas con diferentes caudales que permitieron determinar la dosis óptima, correspondiente 
a una concentración de 4 ppm de ozono en el agua de riego.

Se empleó el método experimental, las plantas fueron cultivadas en campo, se inocularon esporas del hongo Mycosphaerella fijiensis, agente causal de la Sigatoka negra, con la finalidad de asegurar la infestación del hongo en las plantas de banano de la muestra bajo estudio.

La obtención de inóculo del hongo se realizó a partir de hojas infectadas con Sigatoka negra de una plantación contaminada, que contribuya al crecimiento de la infección para su control con el uso del riego ionizado.

\section{DISEÑO DE LA INVESTIGACIÓN}

Se analizaron variables no controlables como clima, tipo de suelo, manejo que fertilización y podas sanitarias, para la selección de plantas que cumplieran con las siguientes características:

- Altura promedio entre $1.5 \mathrm{~m}$. y $2.0 \mathrm{~m}$,

- Mínimo de 9 hojas,

- Plantas distribuidas en una área de $1 / 2$ ha.

Las plantas bajo estudio fueron marcadas y numeradas para impedir su manipulación durante el proceso de evaluación.

El Índice de Severidad (IS), fue calculado mediante la fórmula de Towsend y Heuberguer (Orjeda, 1998).

\section{IS $=\Sigma \mathbf{n} . \mathbf{b} /(\mathbf{N}-\mathbf{1}) \mathrm{T} .100$}

IS= Indice de severidad (en \%)

$\mathrm{n}=$ Número de hojas en cada grado.

$\mathrm{b}=$ Grado en la escala.

$\mathrm{N}=$ Número de grados empleados en la escala. $\mathrm{T}=$ Número total de hojas evaluadas.

TÉCNICAS DE RECOGIDA DE DATOS

La recolección de datos se realizó en los campos de la hacienda Comargara en Jujan, provincia del Guayas, y en la hacienda Florida en Baba, provincia de Los Ríos, colectando hojas afectadas con Sigatoka de la plantación. Para la identificación de la infección se realizaron observaciones en el estéreo microscopio y en el microscopio con cámara. Se prepararon placas portaobjetos con las esporas seleccionadas, las cuales se identificaron y guardaron debidamente. Asimismo, se conformó un archivo fotográfico.

Para evaluar la toxicidad se extrajeron hojas de plantas de banano. El número de esporas en suelo y sustrato se determinó a través del conteo directo de acuerdo al método de Stover.

\begin{tabular}{|ll}
\hline GRADO & DESCRIPCIÓN DEL DAÑO EN LA HOJA \\
\hline 1 & Hasta $10 \%$ manchas por hojas \\
\hline 2 & Menos del 5\% del área foliar enferma. \\
\hline 3 & De 6 a $15 \%$ del área foliar enferma. \\
\hline 4 & De 16 a 33\% del área foliar enferma. \\
\hline 5 & De 34 a $50 \%$ del área foliar enferma. \\
\hline 6 & Más del 50\% del área foliar enferma. \\
\hline
\end{tabular}

DATOS A EVALUAR

Datos agronómicos (evaluados cada 15 días)

- Altura de la planta.

- Diámetro del pseudotallo.

- Número de hojas totales.

- Emisión foliar.

DATOS SANITARIOS

- Severidad de la enfermedad de acuerdo a la escala de Stover.

DATOS DE PRODUCCIÓN

- Peso del racimo

- Número de manos

- Número de clúster

RESULTADOS Y DISCUSIÓN ALTURA DE LA PLANTA

Se midió la altura de planta cada 15 días hasta los 90 días; no existen diferencias significativas del testigo con el resto de los tratamientos, tanto para el ensayo de Jujan como para el ensayo de Baba. (Figura 1)

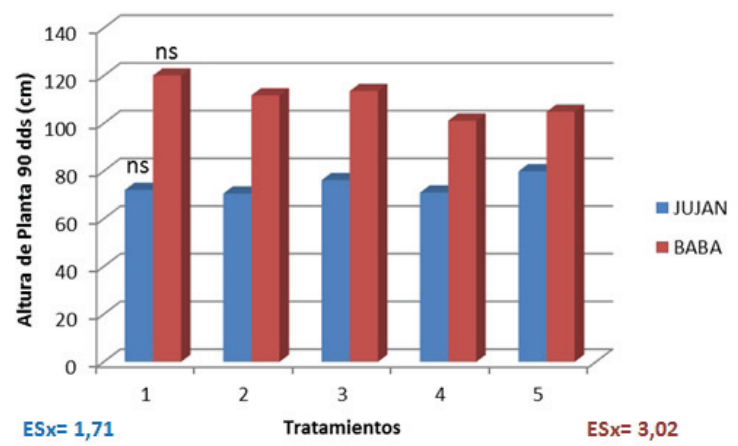

Figura 1. Altura de planta a los $90 \mathrm{dds}$ (cm) ensayo Jujan y Baba. Letras distintas indican diferencias significativas $(p<=0,05$

DIÁMETRO DEL PSEUDOTALLO

Los resultados obtenidos en los dos ensayos no presentan diferencias significativas; en Baba se observó un mayor diámetro de fuste al ser plantas provenientes de cepas mientras que en Jujan las plantas provenían de meristemas. Los 
sistemas de riego y los programas de fertilización fueron los mismos en ambos ensayos, que concuerda con los resultados obtenidos por (Rodríguez-Gaviria \& Cayón, 2008).( Figura 2)

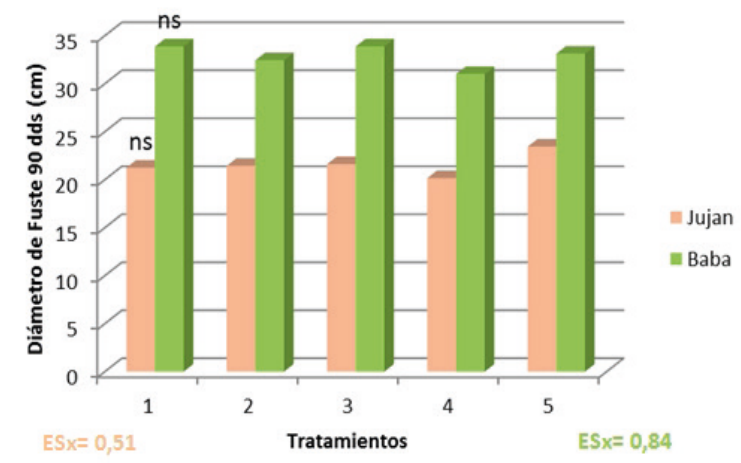

Figura 2. Diámetro de pseudotallo a los 90 dds (cm). Ensayo Baba y Jujan.Letras distintas indican diferencias significativas $(p<=0,05)$

NÚMERO DE HOJAS TOTALES

Los promedios obtenidos no presentan diferencias estadísticas, tanto para el ensayo de Jujan, como para el ensayo en Baba. (Figura 3)

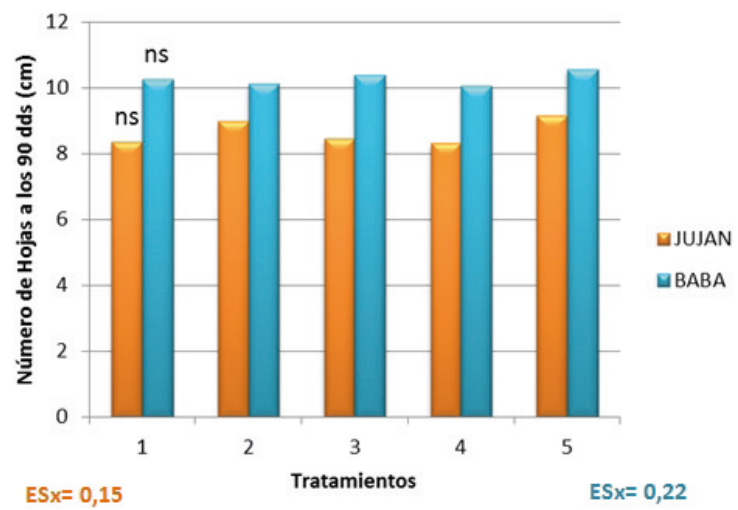

Figura 3. Número de hojas a los 90 dds (cm). Ensayo: jujan y baba. Letras distintas indican diferencias significativas $(p<=0,05)$

EMISIÓN FOLIAR

La emisión foliar se la evaluó con un seguimiento de una hoja cada 7días (Figura 4).

Se pudo comprobar el efecto positivo del uso del ozono disuelto en agua para el control y manejo de la enfermedad, con el fin de decidir y poder recomendar a los pequeños, medianos y grandes productores de banano, las mejores opciones y alternativas para combatir la Sigatoka negra. Según los resultados que se dan en la tabla 1, la cantidad de hojas por planta $(\mathrm{H} / \mathrm{P})$ de floración a cosecha osciló entre 13,25 hojas hasta un máximo de 14,75 hojas en el tratamiento 2 .

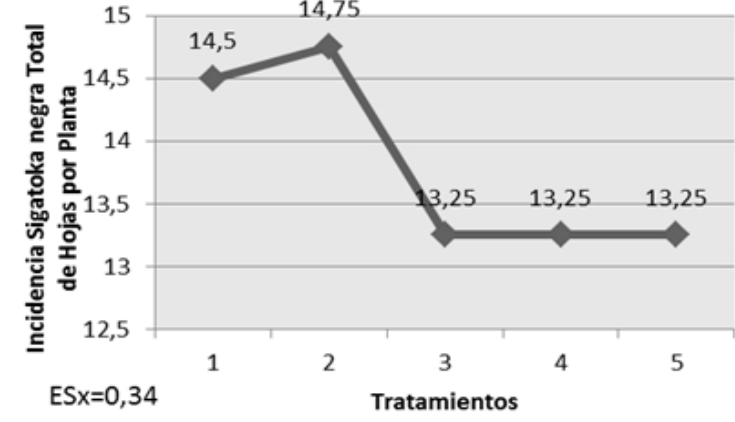

Figura 4. Número de hojas por planta en ensayo, Jujan 2014.

Al comienzo de las evaluaciones en la semana 1, se contó con un mínimo de 3,8 hojas sanas o partes de ellas hasta la semana 35, donde se llegó a un máximo de 14 hojas, en el tratamiento con frecuencia 4. Es importante mencionar que en el testigo absoluto, donde no se aplicó ozono, se llegó al final con 8 hojas, que corresponde al mínimo permisible de hojas para cosechar un fruto para exportación. Con respecto a la variable hoja más joven enferma (HMJE), en la mayoría de los tratamientos evaluados se mantuvo constante, es decir los primeros síntomas comenzaron a partir de la hoja número 4; salvo en los testigos, la enfermedad se manifestó en las hojas más jóvenes, la, número 2 y 3.

El comportamiento de la variable PPI (porcentaje ponderado de infección) resultó más irregular; comenzó con un $0,25 \%$ en el tratamiento con frecuencia 2 , hasta $1,38 \%$, que es el valor máximo, en el tratamiento del testigo absoluto, lo que se demuestra en la Figura 5. Si bien esos porcentajes son realmente bajos, ya que todos se encuentran en el grado 2 , $(<5 \%)$ de la escala de Stover, si reflejan el efecto positivo del ozono en agua con las diferentes frecuencias utilizadas.

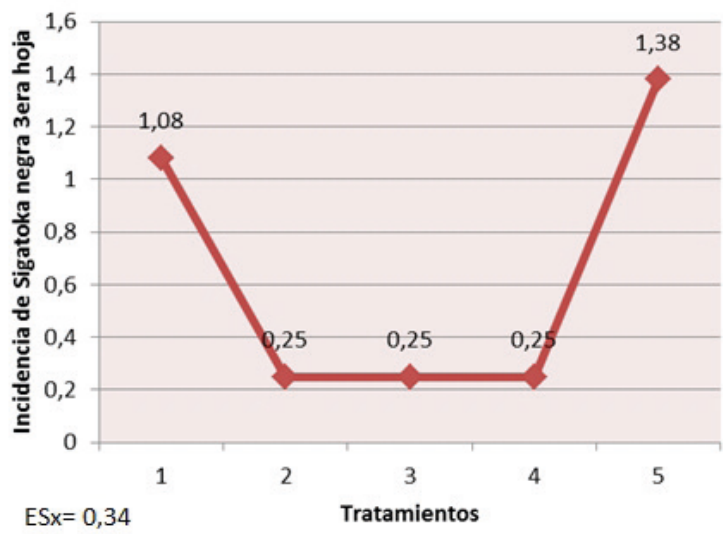

Figura 5. Índice del porcentaje ponderado de infección en ensayo Jujan 2014. 
Se puede considerar que las frecuencias, evaluadas en promedio, tuvieron un comportamiento muy similar en relación con el testigo absoluto. La Figura 5 presenta la dispersión del efecto de los tratamientos durante las 35 semanas de evaluación en la variable PPI, la cual se ubica entre 0,25 hasta $1,38 \%$, comparados con un testigo absoluto.

La dispersión muestra una tendencia a subir al inicio de la evaluación con descensos y altos durante las 35 semanas de evaluación; todos los tratamientos se mostraron muy dispersos en el tiempo.

Se puede asegurar que el ozono disuelto en agua con las frecuencias estudiadas entre 2 y 8 días, pueden realizar un buen control de la enfermedad; no provoca resistencia de parte del hongo a la dosis de ozono utilizada y, especialmente, no se han presentado efectos negativos en la biodiversidad, ni efectos fitotóxicos en las plantas de banano. El costo de esta metodología ha logrado reducir el costo de aplicación de fungicidas químicos en un $40 \%$ menos lo que significa que pueden ser utilizados con una buena rentabilidad por los productores de banano en el combate de la Sigatoka negra a nivel de finca.

TABLA 1. DATOS METEOROLÓGICOS HDA. COMARGARA, CANTÓN JUJAN, PROVINCIA DEL GUAYAS, 2014.
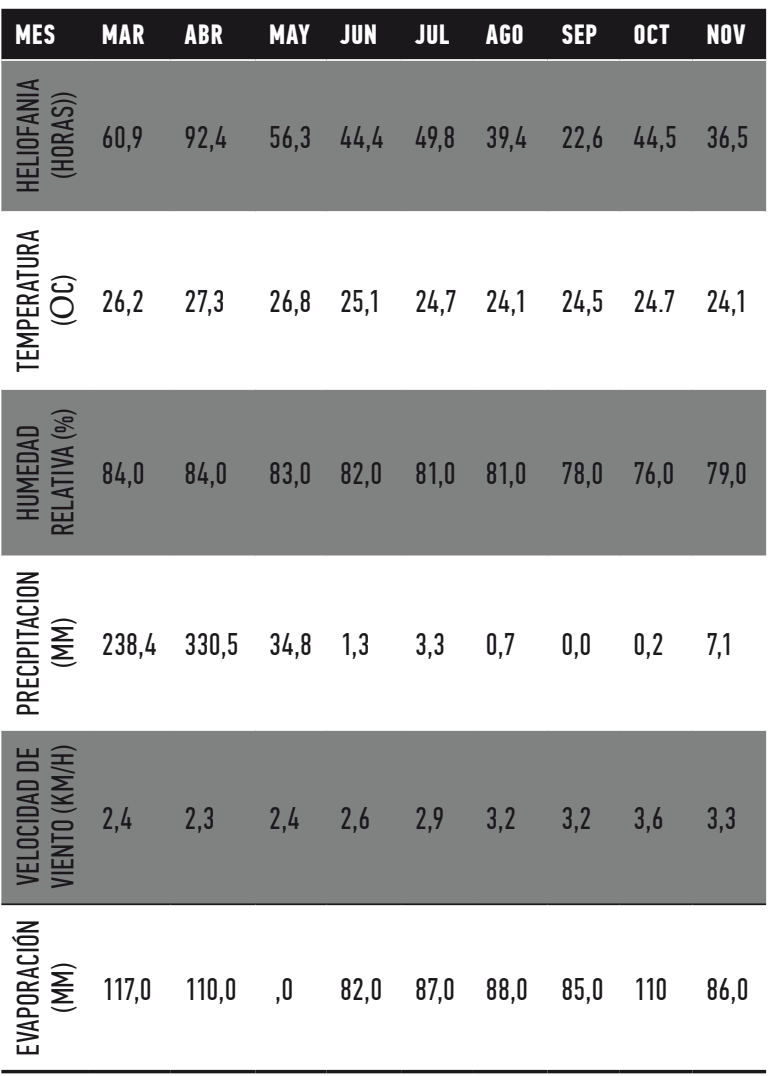

Fuente: Instituto de Meteorología e Hidrología (INAMHI) 2014
La zona de Jujan donde se realizó el ensayo tiene una incidencia baja de la Sigatoka negra, debido a las condiciones edafoclimáticas de la zona (Tablas 1 y 2), sin embargo no se reportó ningún tipo de resistencia al hongo como la creada a los fungicidas químicos.

Es necesario considerar que los factores climáticos presentes durante el periodo de evaluación, así como la influencia de barreras rompevientos naturales existentes, pudieron tener alguna incidencia en la disminución de la enfermedad.

TABLA 2. RESULTADOS DEL USO DE AGUA OZONIZADA PARA EL CONTROL DE LA SIGATOKA NEGRA EN BANANO. ENSAYO EN JUJAN.

\begin{tabular}{|c|c|c|c|c|c|}
\hline \multirow[b]{2}{*}{ TRATAMIENTO } & \multirow[b]{2}{*}{ FRECUENCIAS } & \multicolumn{4}{|c|}{ VARIABLES EVALUADAS } \\
\hline & & $\mathrm{H} / \mathrm{P}$ & HMJE & $\begin{array}{l}\text { PPI } 3^{\circ} \\
\text { HOJA }\end{array}$ & $\begin{array}{l}\mathrm{PPI} 4^{\circ} \\
\text { HOJA }\end{array}$ \\
\hline 1 & 2 días & $14,50 \mathrm{~ns}$ & 12,25 & ns1,08 & 1,17 \\
\hline 2 & 4 días & 14,75 & 11,50 & 0,25 & 1,17 \\
\hline 3 & 6 días & 13,25 & 11,50 & 0,25 & 1,34 \\
\hline 4 & 8 días & 13,25 & 10,75 & 0,25 & 1,42 \\
\hline 5 & Testigo & 13,25 & 9,75 & 1,38 & 1,42 \\
\hline
\end{tabular}

La representación gráfica de las frecuencias y dosis utilizadas, reflejan una dispersión más compacta, mostrando una curva sigmoidea a lo largo de las 35 semanas de evaluación y concentrándose entre 0,25 a 1,8\% de infección; este porcentaje se ubica según la escala de Stover en el grado 2, hasta un $5 \%$ del área de la hoja con manchas (Guzmán, Jiménez y Rengifo, 2001).

El comportamiento es muy similar para todos los tratamientos comparados con el testigo absoluto.

Se considera que el avance es muy rápido, pues la hoja más joven enferma es, en todos los tratamientos, la número 3; el grado ponderado de infección es bajo relativamente y puede ser controlado o manejado utilizando el agua ozonificada pues no existen diferencias significativas entre las frecuencias de aplicación evaluadas en este estudio.

Estos resultados se complementan con los obtenidos por (Aguirre, 2012) los actores recomiendan el uso de $4 \mathrm{ml}$ de ozono/ha sin efectos fitotóxicos sobre la planta y un buen control de la Sigatoka negra.

Comparaciones entre tratamientos con diferentes frecuencias de aplicación y el testigo absoluto

Se realizaron diferentes comparaciones entre los tratamientos y las variables evaluadas para poder discriminar con propiedad el efecto del ozono disuelto en agua en el combate de la Sigatoka negra.

La comparación de las hojas por planta (HP) en los tratamientos con frecuencia de 2, 4, 6, y 8 días 
y el testigo absoluto se presentan en la Figura 6. Los tratamientos con las diferentes frecuencias comparados con el testigo absoluto muestran que fueron más eficientes en mantener la sanidad de las hojas sanas hasta un número de 14 hojas en promedio a la cosecha, mientras que el testigo, apenas logró como máximo 10 hojas sanas o parte de ellas a la cosecha.

El efecto del ozono disuelto en agua, por su modo de acción al oxidar las membranas del hongo Mycosphaerella, garantizan una mejor cobertura y un efecto residual de más largo alcance en relación con el control químico del hongo, considerando además la reducción de un $40 \%$ en su costo de aplicación, lo que lo hace muy viable para el cultivo del banano en pequeñas, medianas y grandes plantaciones.

En la zona de Baba el comportamiento del uso del ozono en agua con la dosis utilizada, tuvo el mismo comportamiento en las variables evaluadas. En la variable HMJE, el testigo absoluto muestra la infección a partir de la hoja 3 a 5 predominando entre la 3 y la 4, (Figura 6 ) mientras que en los tratamientos con frecuencia variada la hoja más joven enferma se localiza a partir de la 4,0 a la hoja 9, 10, predominando entre la hoja 5 a 7.

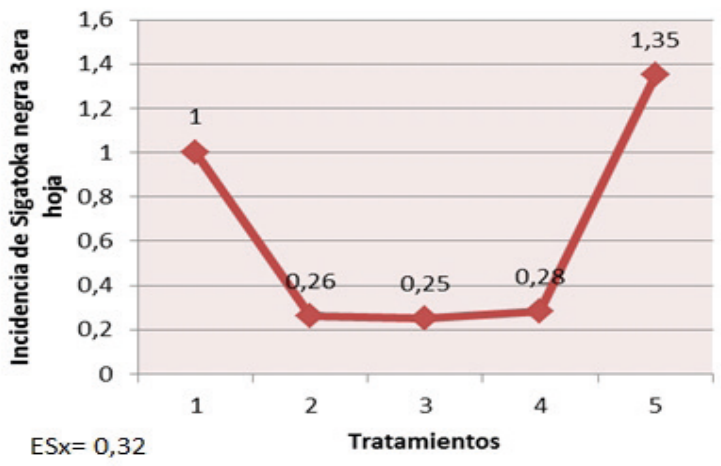

Figura 6. Índice del porcentaje ponderado de infección en ensayo de Baba 2014.

Esto muestra la eficacia del uso del ozono en el control de la enfermedad. Durante el periodo de evaluación, la mayor incidencia se localizó entre las semanas 1 a la 20. A partir de la 20 semana, la hoja más joven en el tratamiento 4, se mantuvo en la hoja 4 y 5; el testigo absoluto se mantuvo entre la 3 y la hoja 4, respectivamente.

En relación al PPI (Figura 7), en los tratamientos donde se utilizó las frecuencias de 2 y 4 días se localiza entre los niveles más bajos, menores de $1,04 \%$ hasta $1,37 \%$, predominando el 1,0\% durante las primeras semanas hasta la semana 20, después de la cual se mantienen muy similares, ubicados entre el 1,19 y 1,37\%, lo que corresponde al grado 2 de la escala de Stover.

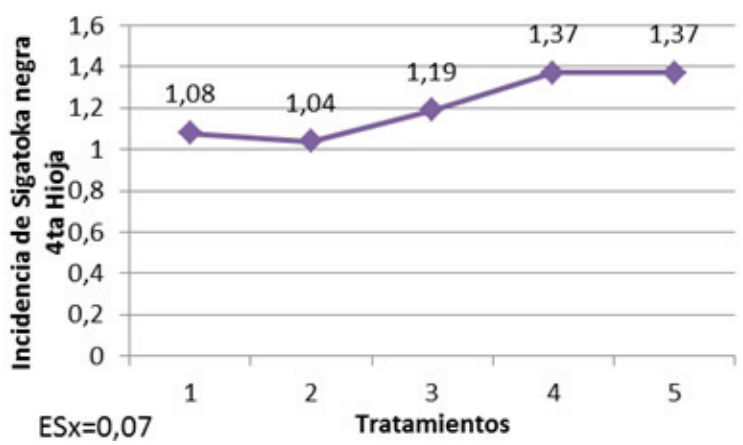

Figura 7. Índice de infección de Sigatoka negra en 4 ta hoja.

Resultado de la prueba del uso del ozono en agua en el combate de Sigatoka negra en banano, durante 35 semanas de evaluación.

TABLA 3. DATOS METEOROLÓGICOS HDA. FLORIDA, BABA, PROVINCIA DE LOS RÍOS, 2014.
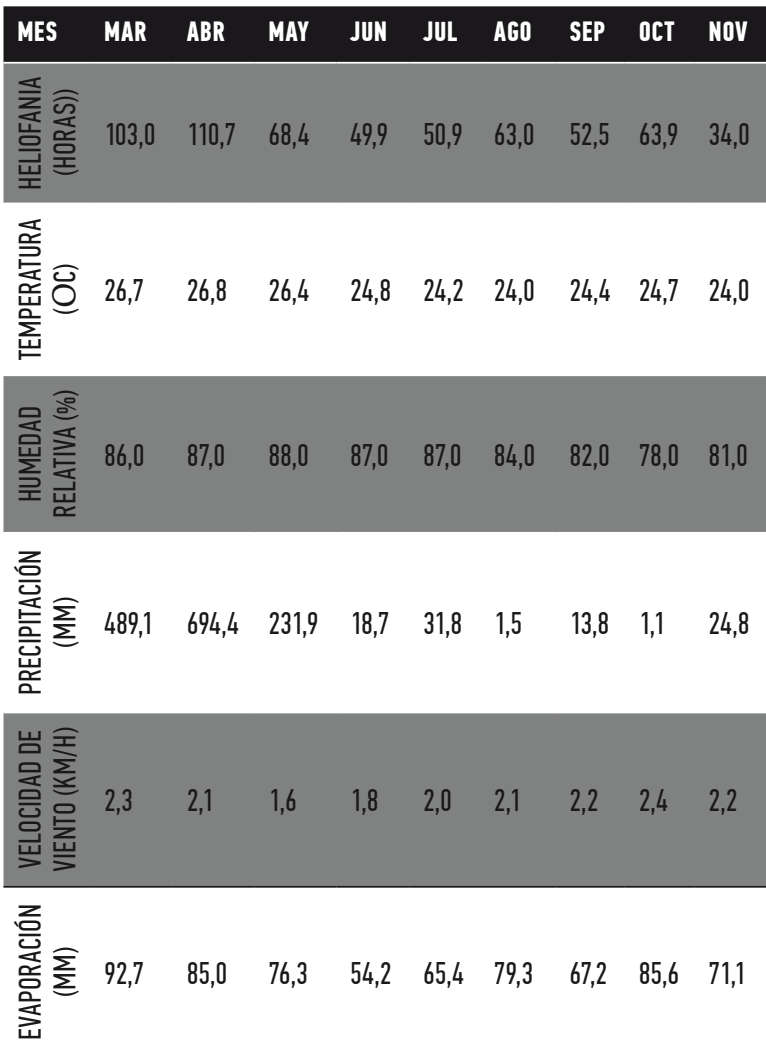

Fuente: Instituto de Meteorología e Hidrología (INAMHI) 2014

Antes del proceso de empaque del banano, se toman los datos del peso del racimo, el número de manos por racimo, número de clúster por racimo, peso de clúster y peso de clúster de rechazo; estos datos son de mucha importancia para realizar los análisis y cuadros estadísticos de los resultados del proyecto. 
TABLA 4. RESULTADOS DEL USO DE AGUA OZONIZADA PARA EL CONTROL DE LA SIGATOKA NEGRA EN BANANO. ENSAYO EN BABA.

\begin{tabular}{|c|c|c|c|c|c|}
\hline \multirow[b]{2}{*}{ TRATAMIENTO } & \multirow[b]{2}{*}{ FRECUENCIAS } & \multicolumn{4}{|c|}{ VARIABLES EVALUADAS } \\
\hline & & $\mathrm{H} / \mathrm{P}$ & HMJE & $\begin{array}{l}\text { PPI } 3^{\circ} \\
\text { HOJA }\end{array}$ & $\begin{array}{l}\text { PPI } 4^{\circ} \\
\text { HOJA }\end{array}$ \\
\hline 1 & 2 días & 13,53 NS & $11,53 \mathrm{NS}$ & $1 \mathrm{NS}$ & 1,08 NS \\
\hline 2 & 4 días & 14,38 & 10,59 & 0,26 & 1,04 \\
\hline 3 & 6 días & 14,15 & 10,71 & 0,25 & 1,19 \\
\hline 4 & 8 días & 14,03 & 10,30 & 0,28 & 1,37 \\
\hline 5 & Testigo & 13,88 & 10,35 & 1,35 & 1,37 \\
\hline
\end{tabular}

Finalmente, en las labores de cosecha se pudo observar que en los tratamientos 2 y 3, se obtuvieron el pesaje más alto con 70 libras; en el tratamiento 3 con 25 clúster y un peso de 51,5 libras ha sido el valor más alto en el proyecto, tomando como referencia los requisitos de exportación ya que el peso de una caja es de 43 libras con un número de 18 a 19 clúster.

DATOS DE PRODUCCIÓN PESO DE RACIMO

Los promedios obtenidos en el peso de los racimos de los diferentes tratamientos no presentaron diferencias estadísticas, sin embargo en el ensayo de Baba se encontró diferencia numérica entre el testigo y los tratamientos 1,3 y 4 debido probablemente a la influencia de ozono sobre el suelo, que también resulta un elemento con un poder oxidante de los nutrientes del suelo, que se encuentran unidos a las micelas coloidales del suelo, provocando su disponibilidad para las plantas, lo que concuerda con lo manifestado por (Ramírez y Sáinz (2012).) donde indican que el Ozono posee un poder oxigenante mayor que el del oxígeno normal y por ello mejora el proceso respiratorio a nivel celular.

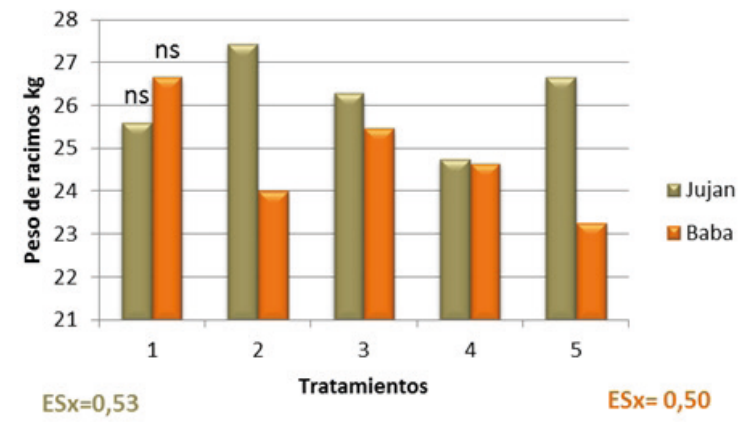

Figura 8. Peso de racimos (kg) a la cosecha. Ensayo Jujan y Baba. Letras distintas indican diferencias significativas $(p<=0,05)$

NÚMERO DE MANOS

En lo que respecta al número de manos los resultados siguieron el mismo comportamiento de no presentar diferencias estadísticas, sin embargo se encontró diferencia numérica, donde los promedios de Jujan presentaron un mayor número de manos, lo que se muestra en la Figura 9 .

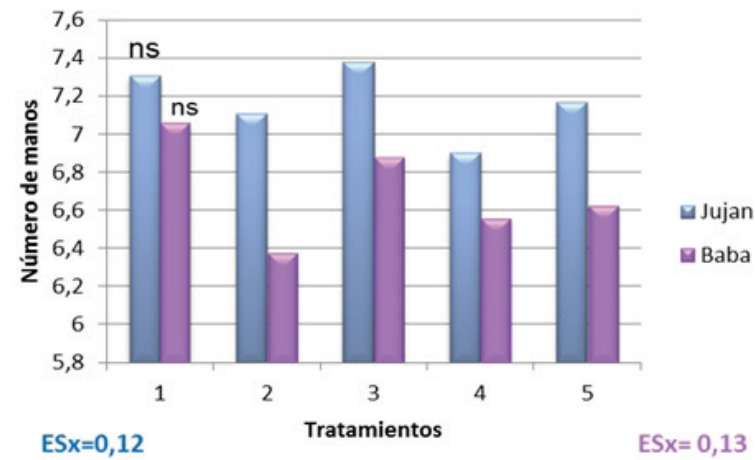

Figura 9. Número de manos por racimo a la cosecha. Ensayo Jujan y Baba letras distintas indican diferencias significativas $(p<=0,05)$

NÚMERO DE CLÚSTER

Los resultados obtenidos de los promedios de número de clúster no presentan diferencias estadísticas tanto para el ensayo de Jujan como para el de Baba; esto se debe probablemente al buen control ejercido de la Sigatoka negra por la aplicación del ozono en agua, el cual además de su efecto sobre el hongo, ayuda en los procesos de oxidación celular, produciendo un mayor desarrollo de la fruta, lo que concuerda con los resultados obtenidos por Aguirre (2012).

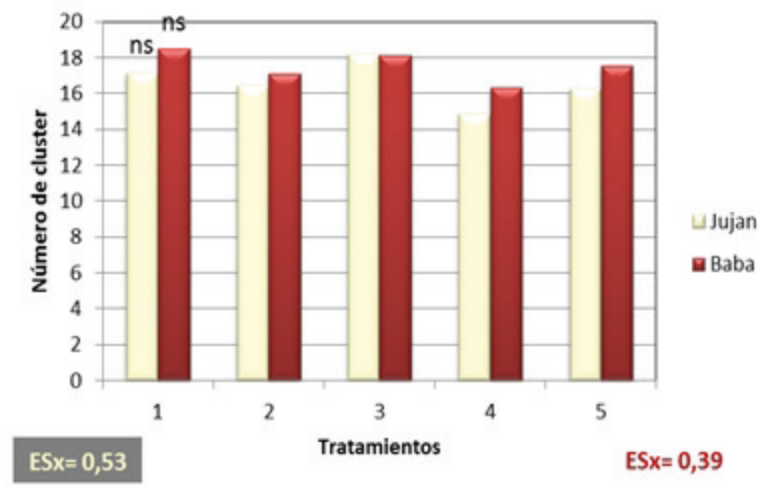

Figura 10. Número de clúster por racimo a la cosecha. Ensayo Jujan y Baba letras distintas indican diferencias significativas $(p<=0,05)$

CONCLUSIONES

Se diseñó un equipo de campo para la producción de ozono, basado en la técnica de descarga eléctrica

Se determinó la concentración de ozono óptima y la frecuencia de aplicación para un control efectivo de Micosphaerella fijiensis en condiciones de campo, en las dos provincias estudiadas. 
Los mejores tratamientos son aquellos donde se usó el ozono disuelto en agua con frecuencia de 4, 6 y 8 días con una concentración de $4 \mathrm{mg} / \mathrm{L}$ de ozono.

El ozono disuelto en agua posee propiedades fungicidas al demostrarse la disminución de la severidad de la enfermedad durante el período de tiempo en las cuales se realizaron las evaluaciones en campo.

\section{REFERENCIAS BIBLIOGRÁFICAS}

Aguirre,C. (2012). Estudio de la efectividad del uso del ozono como fitosanitario en banano. Memoria Técnica. Itagra.ct. Pueblo Viejo, Los Rios, Ecuador.

Arencibia, J., Leyva, Y., Collymore, A. \& Araujo,J. (2006).. Producción científica sobre aplicaciones terapéuticas del ozono en el Web ofScience. ACIMED online14(1) Disponible en: <http://scielo.sld.cu/scielo.php?script=sci_ arttext\&pid=S1024-94352006000100007\&lng= es\&nrm=iso>. ISSN 1024-9435.

Beutelspacher, S. y Calderón, J. (2005). Diseño y construcción de un generador de ozono para aplicaciones de purificación de agua. Tesis inédita de maestría. Centro Nacional de Investigación y Desarrollo Tecnológico. Cuernavaca Morelos, México.

Escudero M. C y Rondón, E. C. (1996). Integrate management experiences with black sigatoka (Mycosphaerella fijiensis) and non-systemic fungicides in Soconusco, Chiapas, México. 53p. In XI Meeting ACORBAT. Santo Domingo, República Dominicana (Resumen).

Guzmán, P., Jiménez, I. y Rengifo, C. (2001). Transmisibilidad de hongos de la semilla a la planta de arroz. Arroz 50 (433):25-29

Guzmán, M; Villalta, R. (2006). Efecto del método de control de malezas sobre el desarrollo de la Sigatoka negra y la producción del banano (Musa AAA, cv. Grande naine). CORBANA 33(60):50-52.

Martínez, I., Villalta, R., Soto, E., Murillo,G. y Guzmán, M.(2011). Manejo de la Sigatoka negra en el cultivo del banano. CORBANA. Hoja Divulgativa n.o 2-2011. Recuperado de http:// www.infoagro.net/programas/Ambiente/ pages/adaptacion/casos/Sigatoka.pdf

Martínez, I. y Guzmán, M. (2010). Eficacia biológica en el combate de la Sigatoka negra de la aplicación con fungicidas con motobomba de espalda equipada con un cabezal de conversión electrostática. Informe Anual 2009. Dirección de Investigaciones CORBANA (Corporación Bananera Nacional, CR).San José, CR.
Orjeda, G. (1998) Evaluación de la resistencia de los bananos a las enfermedades de Sigatoka negra y marchitamiento por Fusarium. Guías técnicas INIBAP 3. IPGRI, Roma, Italia; Red Internacional para el mejoramiento del banano y el plátano, Montpellier, Francia

Orozco - Santos, M. (1998). Manejo Integrado de Sigatoka Negra del plátano. Campo Experimental Tecoman, MEX. INEFAP. 45-55.

Orozco - Santos M., Farías L., Manzo, J., Sánchez. G y Guzmán, S. (2001). La Sigatoka Negra (Mycosphaerella fijiensis Morelet) in México. Infomusa La revista internacional sobre banano et plátano 10 (1): 33p

Patiño, L., Bustamante, E., Salazar, L. (2007). Efecto de sustratos foliares sobre la sigatoka negra (Mycosphaerella fijiensis Morelet) en banano y plátano. Revista de agricultura técnica 67(4):437-445.

Ramírez, J. y Sáinz R. (2012). El ozono en la agricultura y el bienestar. Universidad Autónoma de Sinaloa, México DF.

Riveros, AS. y Lepoivre, P. (1998). Mecanismos de defensa asociados con la resistencia total en lainteracción $M$. fijiensis - Musa. In Memorias: I Seminario Internacional sobre la producción de plátano. Armenia, Quindío, CO. p 56-62.

Rodríguez-Gaviria, A. y Cayón, G. (2008). Efecto de Mycosphaerella fijiensis sobre la fisiología de la hoja de banano. Agronomía Colombiana 26(2): 256-265.

Stover, R.H. (1980). Sigatoka leaf spot of banana and plantains. Plant disease 64: 750-756

Tumbaco, J. y Jiménez, M. (2011). Evaluación del efecto sobre Sigatoka negra, en hojas separadas de banano, Cavendish (variedad Williams) del extracto de Melaleuca alternifolia en tres zonas del litoral Ecuatoriano. Tesis inédita de grado. Escuela Superior Politécnica del Litoral. Guayaquil, Ecuador.

Zuluaga, C., Patiño, L. y Collazos, J. (2007). Integración de inducción de resistencia con bacterias quitinolíticas en el control de la sigatoka negra (Mycosphaerella fijiensis Morelet) en banano. Rev.Fac.Nal.Agr. Medellín. Colombia 60 (2): 3891-3893. 